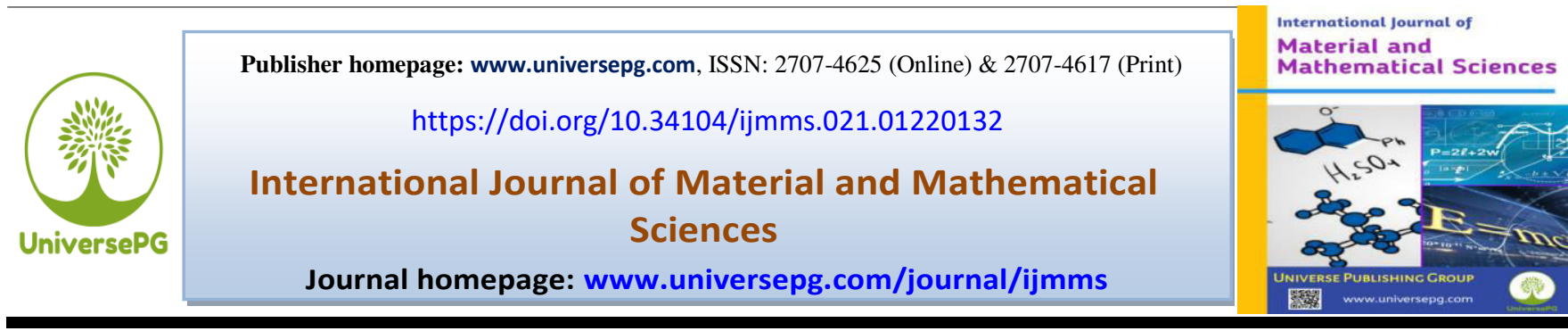

\title{
Investigation of the Effect of 3d TM-TM Atom Co-Doped in Graphene Nanosheet: DFT Based Calculations
}

\author{
Maliha Nishat ${ }^{1}$, Rojina Islam ${ }^{2}$, and Md. Atikur Rahman ${ }^{1}$ * \\ ${ }^{1}$ Department of Physics, Pabna University of Science and Technology, Pabna-6600, Bangladesh; ${ }^{2}$ Deptarment of Physics, \\ Jahangirnagar University, Savar, Dhaka-1342, Bangladesh. \\ *Correspondence: atik0707phy@gmail.com (Md. Atikur Rahman, Department of Physics, Pabna University of Science and \\ Technology, Pabna-6600, Bangladesh).
}

\begin{abstract}
Graphene, an interesting 2D system has a rare electronic structure of two inverted Dirac cones touching at a single point, with great electron mobility and promising microelectronics applications. In the present article, a theoretical investigation has been performed on the structural, electronic and magnetic properties of pristine graphene nanosheet and also the effect of $3 \mathrm{~d}$ transition metal (TM) co-doped in graphene nanosheet within the density functional theory framework. $3 \mathrm{~d}$ TM is categorized into two groups: $\mathrm{Cr}$ - group (Cr-Cr, $\mathrm{Cr}-\mathrm{Mn}$, and $\mathrm{Cr}-$ $\mathrm{Fe}$ ) and $\mathrm{Ni}$-group (Ni-Cr, Ni-Ti, Ni-Mn). After co-doping TM atoms on graphene, it still holds its planar shape which refers to the stability of these co-doped graphene nanosheets. This is also confirmed by the increasing bond length of carbon and TM atoms on graphene nanosheets. Highest zero-point energies have been found of $12049.24 \mathrm{eV}$ and $-10936.87 \mathrm{eV}$ respectively for $\mathrm{Cr}-\mathrm{Cr}$ and $\mathrm{Ni}-\mathrm{Cr}$ co-doped graphene nanosheet. According to Mulliken's charge and electron density differences, all the TM atoms can act as electron donors while the graphene nanosheet is electron acceptor. All the TMs co-doped graphene nanosheet show metallic behavior in terms of band structures and DOS plots except Ti-Ni which has shown a little band gap. In terms of electronic properties, $\mathrm{Cr}-\mathrm{Cr}$ and $\mathrm{Ni}-\mathrm{Cr}$ co-doped graphene nanosheets are found most stable among the other studied systems and they can exhibit magnetic behavior as there is a variation in their up and down spin as shown in spin polarized DOS. That's why they are beneficial to the application of various magnetic devices as well as sectors. Besides Cr-group co-doped graphene nanosheet can exhibit better magnetic properties than Ni-group.
\end{abstract}

Keywords: Graphene, Nanosheet, Co-doped, DFT Calculations, Transition metal, and Magnetic properties.

\section{INTRODUCTION:}

For the past decades, graphene which is a one-atomthick planar sheet of $\mathrm{sp}^{2}$ hybridized carbon atoms closely packed in a honeycomb lattice structure, has caught significant consideration to be utilized as a cutting edge electronic material. This is possible because of its remarkable properties including great current density, chemical inertness, optical transmission, huge thermal conductivity, ballistic transport and marvelous hydrophobicity at nano scale range (Geim et al., 2008), UniversePG I www.universepg.com
(Chen et al., 2008). Micro-mechanical cleavage was the method used for first graphene extraction from graphite and this method permitted simple fabrication of first-rate graphene crystallites and further prompted tremendous experimental activities (Clintock, 2012).

Fundamentally graphene is a huge aromatic macromolecule with no band gap and it can absorbs electromagnetic energy homogeneously over the electromagnetic spectrum, from infrared through visible to 
ultraviolet (Avouris, 2010). As a whole, great surface area renders to improve its reactivity. Again, since graphene has plentiful surface area for reactions to occur, it is likewise of attention in aspects of medicine, biology and bioengineering for the reason it is suitable for a biologically well-suited electrode and drug delivery material (Vanesa et al., 2012).

Both theoretical (Chen et al., 1997; Menon et al., 2000) and experimental (Binns et al., 1996; Nagao et al., 1998) investigations have been done to understand the behavior of TM atoms when it interacted with graphite or a graphene. These investigations demonstrate that carbon' $\mathrm{sp}_{\mathrm{z}}$ orbitals hybridize intensely with the ' $\mathrm{d}$ ' orbitals of the TM atoms. Half-metallic systems have been found by the interaction process of magnetic atoms with nanotubes that are of interest for nanomagnets and spintronics devices (Alphenaar et al., 2001). The great moments of Fe and Co doped structures and the half metallic behavior for specific singleatom Co doped structures could be suitable for magnetic device and spintronics functions (Yagi et al., 2004). It has also investigated that doping of the edges of the similar graphene nanoribbon with various atoms, from s-type to d-type transition metals; we can achieve numerous electronic and magnetic properties. In fact, different dopants doped the similar ribbon which can be insulator, semiconductor, metal, ferro-magnetic and anti ferromagnetic. Doping in 3d transi-tion metals can be supplied systems with FM or AF circumstances at the edges. Doping of the edge with little densities of $\mathrm{Fe}$ or Mn can cause in half-metallic or half-semiconductor ribbon. Consequently, graphene nanoribbons give an extensive range of promising electronic and magnetic phenomenon like similar ribbon structure but various dopant TM atoms (Pathi-ranage, 2021; Gorjizadeh et al., 2008).

In our investigation, the structural, electronic and magnetic properties of pristine graphene and $3 \mathrm{~d}$ transition metal co-doped graphene nanosheet have been studied. In this article, focus has been made on the co-doping of $3 \mathrm{~d}$ TM atoms onto graphene nanosheet. We have investigated how these co-doped transition metals (Cr-Cr, Cr-Mn, Cr-Fe, Ni-Ti, Ni-Cr, $\mathrm{Ni}-\mathrm{Fe}$ ) impact different physical properties of $2 \mathrm{D}$ graphene nanosheet.

UniversePG I www.universepg.com

\section{Computational Details}

In this study, we perform spin-polarized ab initio calculations using the Cambridge Serial Total Energy (CASTEP) code (Segall et al., 2002) within the framework of density functional theory (Rajagopal et al., 1973; Kohn \& Sham, 1965). In this code, the KohnSham equations are solved and the wave functions of valence electrons are expanded in a basis set of plane waves with kinetic energy smaller than specified cutoff energy, $\mathrm{E}_{\text {cut }}$. A nonlocal ultra-soft pseudo-potential of the Vanderbilt-type represents the presence of tightly-bound core electrons and also described the electron-ion interaction. The exchange correlation potential is treated within the Perdew-Burke-Ernzerhof, (1996) version of the generalized gradient approximation (PBE-GGA) (Perdew et al., 1996). A super cell contained 56 carbon $(\mathrm{C})$ atoms is considered as the basic model for calculation which is a plane graphene nanosheet. Here $1 \times 3 \times 2$ Monkhorst-Pack grid (Monkhorst \& Pack, 1976) in the Brillouin zone of the unit cell was employed for the geometry optimization so that it can generate an even grid of k-points along the three axes in reciprocal space. We use PBE gradient corrected functional and ultra soft pseudo potentials with plane-wave kinetic energy cutoff energies of 310 $\mathrm{eV}$. Graphene nanosheet is built of perfect geometry with an initial C-C distance of $1.42 \AA$. Geometry optimization has been performed for all investigating nanosheets with the Broyden-Fletcher Goldfarb-Shanno (BFGS) minimization technique, with the flowing ambit for converged structures: energy change per atom less than $1 \times 10^{-5} \mathrm{eV}$, residual force less than 0.03 $\mathrm{eV} / \AA$, stress below $0.05 \mathrm{GPa}$ and the displacement of atoms during the geometry optimization less than $0.001 \AA$.

In the TM co-doped graphene nanosheets, for efficient comparison, every time we have replaced the C20 and C30 atom of the pristine nanosheet by the targeted TMs. Thus, different structural, electronic and magnetic properties of the $3 \mathrm{~d}$ TM co-doped graphene nanosheets were compared and investigated by the processes discussed above.

\section{RESULTS AND DISCUSSION:}

For better understanding, we have divided our study of $3 \mathrm{~d}$ TM co-doped graphene nanosheet into two groups in this section. They are $\mathrm{Cr}$ co-doped (Cr-Cr, Cr-Mn, 
$\mathrm{Cr}-\mathrm{Fe})$ group and $\mathrm{Ni}$ co-doped (Ni-Ti, Ni-Cr, $\mathrm{Ni}-\mathrm{Fe})$ group. Here 3d TMs are Ti, Cr, Mn, Fe, Ni.

\section{Effect of Cr-3d TM co-doping in Graphene Nano- sheet}

\section{Structural Properties}

When a substance is doped by any hetero element its structural properties are changed radically which leaves impact to the geometric and structural properties of the mother substance. Thus structural stability analysis is an important observation when a material is doped by other hetero elements. In this investigation, it is noticed that optimized pristine graphene and $\mathrm{Cr}-\mathrm{Cr}, \mathrm{Cr}-\mathrm{Mn}, \mathrm{Cr}-\mathrm{Fe}$ co-doped graphene nanosheets are perfect planer. Besides, in co-doped structures, bond lengths are distorted around the TM

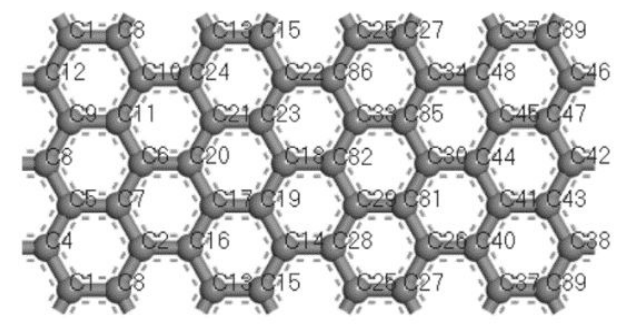

Optimized structure

\section{a. Pristine graphene}

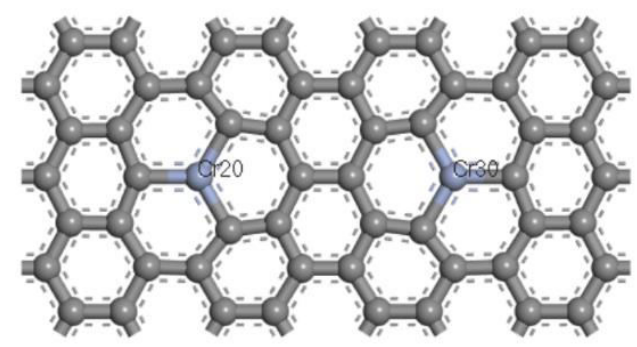

Optimized structure

\section{b. $\mathrm{Cr}$-Cr co-doped graphene nanosheet}

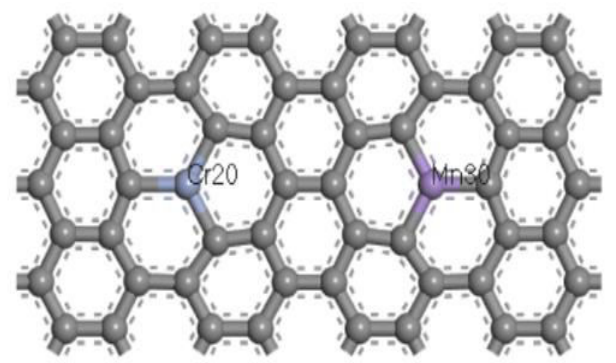

Optimized structure

\section{c. Cr-Mn co-doped graphene nanosheet}

atoms. In the pristine graphene nanosheet, every time we have replaced $\mathrm{C} 20$ by $\mathrm{Cr}$ atom and $\mathrm{C} 30$ is by $\mathrm{Cr}$, $\mathrm{Mn}$ and $\mathrm{Fe}$ atoms respectively. Here, C20 can be considered as TM1 position and the position of $\mathrm{C} 30$ is TM2. The charge transfer is the transference of fractional electronic charge between the complex system which creates an electrostatic attraction in the complex system or molecule. This electrostatic attarction is the root of forming stabilizing force in a complex system and the portion which transfer charge is recognized as electron donor and the receiving entities is known as electron acceptor. The charge transfer occurs due to the difference in the electron density of the constituents of a system i.e. some have excess of electrons and some have lack of electrons.

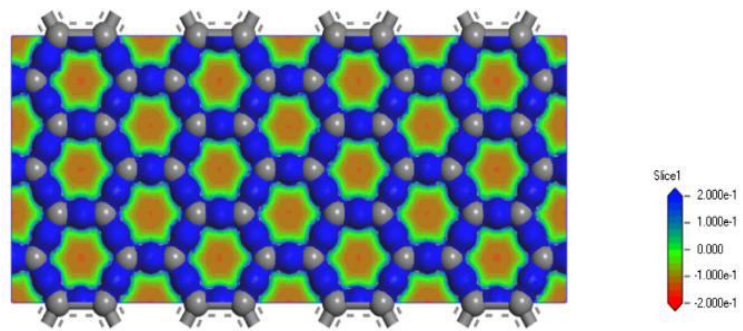

Electron density difference

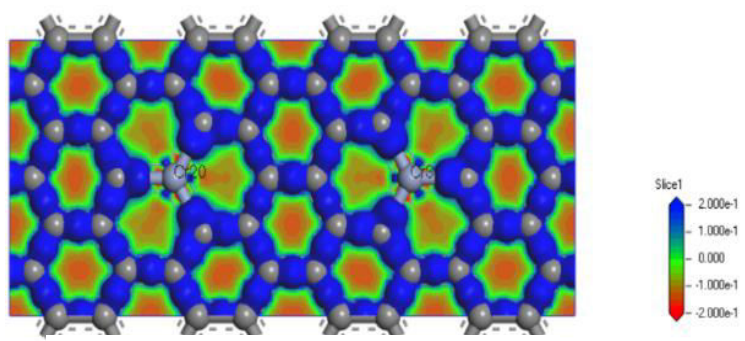

Electron density difference

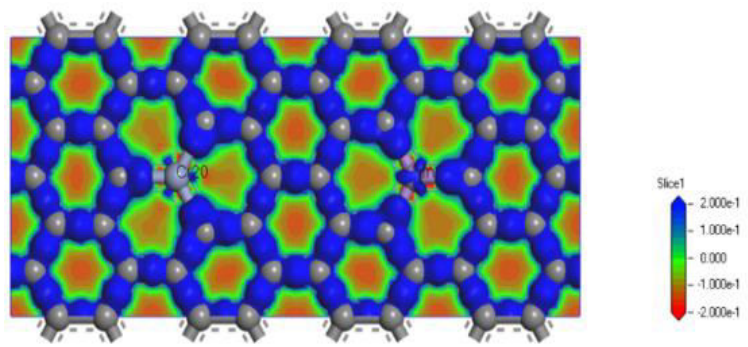

Electron density difference 


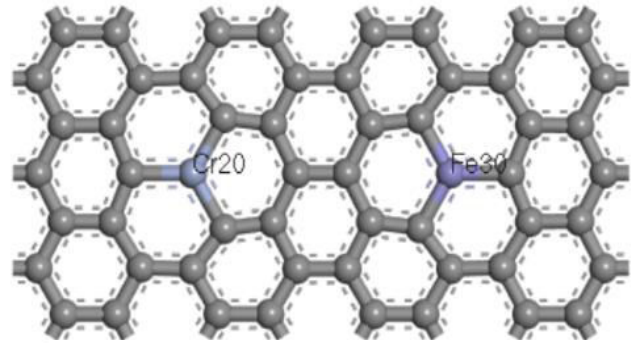

Optimized structure

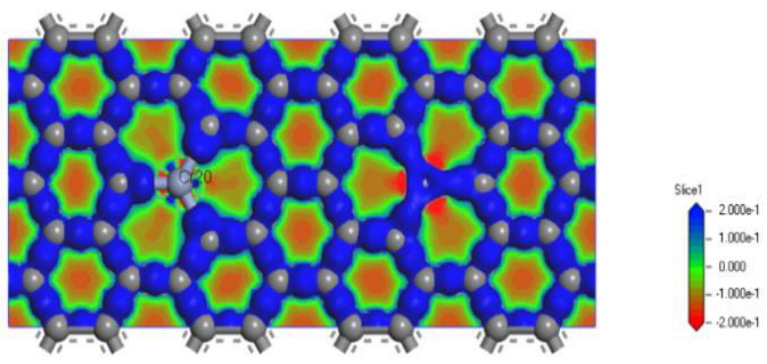

Electron density difference

\section{d. Cr-Fe co-doped graphene nanosheet}

Fig 1: Optimized structure and electron density difference of pristine and $\mathrm{Cr}-\mathrm{Cr}, \mathrm{Cr}-\mathrm{Mn}, \mathrm{Cr}-\mathrm{Fe}$ co-doped graphene nanosheet.

For that reason, charge transfer investigation is a very crucial study for realizing the stability and various structural phenomenon of a nanostructure $(\operatorname{Rad}$ et al., 2016; Rad, 2016; Wegner et al., 2008). We have investigated Mulliken charge distribution using the same level of theory for all the optimized pristine and $\mathrm{Cr}$ group co-doped graphene nanosheet and the value of Mulliken charge transfer between the donors and acceptors are tabulated in Table $\mathbf{1 .}$

Table 1: Data of Mulliken charge transfer for pristine graphene (PG), Cr-Cr, Cr-Mn and $\mathrm{Cr}-\mathrm{Fe}$ co-doped graphene nanosheet.

\begin{tabular}{|c|c|c|c|c|}
\hline Species & $\begin{array}{c}\text { Pristine } \\
\text { graphene }\end{array}$ & $\begin{array}{c}\text { Cr-Cr } \\
\text { (TM1-TM2) }\end{array}$ & $\begin{array}{c}\text { Cr-Mn } \\
\text { (TM1-TM2) }\end{array}$ & $\begin{array}{c}\text { Cr-Fe } \\
\text { (TM1-TM2) }\end{array}$ \\
\hline C6 & 0 & -0.29 & -0.30 & -0.30 \\
\hline C17 & 0 & -0.30 & -0.29 & -0.30 \\
\hline C21 & 0 & -0.30 & -0.29 & -0.30 \\
\hline C31 & 0 & -0.30 & -0.22 & -0.33 \\
\hline C35 & 0 & -0.30 & -0.22 & -0.33 \\
\hline C44 & 0 & -0.29 & -0.22 & -0.33 \\
\hline TM1 & - & 1.70 & 1.78 & 1.69 \\
\hline TM2 & - & 1.70 & 1.34 & 1.80 \\
\hline
\end{tabular}

It is noticed that there is a creation of donor and acceptor within the nanosheet and it is possible as it depends on the electro negativity of the constituents' atoms of the graphene nanosheet. The TMs are acted as electron donor; in our optimized Cr-TM co-doped graphene nanosheet the charges are positive for doped transition metals. Again in Fig 1 each of the 16 colors represents a specific range of the charge density. Here electron enrichment is indicated in blue, white indicates regions with very little change in the electron density and red infers electron depletion. This change of charge transfer modifies stability, reactivity, band gap etc. of the studied pristine graphene nanosheet which makes it a favorable candidate for diverse applications. Bond lengths and atomic distances in the nanosheet are directly dependent on the charge distribution of the system. Bond lengths between the ato$\mathrm{ms}$ are investigated by using the same theory and they are tabulated in Table 2.

From Table 2, it is clear that in pristine graphene C-C distance is constant though the nanosheet which is 1.42 $\AA$. As the atomic radii of the TMs far greater than C atoms in the pristine grap-hene nanosheet, the bond lengths between the $\mathrm{C}$ atoms and TMs are increased. This indicates that TMs are well adsorbed in graphene nanosheet. Again the bond length analysis of the codoped graphene nanosheet shows similar trend for the causes already mentioned in the charge distribution analysis sections. Lattice constants refer to the physical dimension of the unit cells in a crystal lattice and it is an indicator for the measurement of the structural compatibility between different materials. Here, in this study, it is observed that every investigating nanosheets have same lattice constant $(\mathrm{a}=17.04$, $b=7.38, c=16)$ and same bond angles $(\alpha=\beta=\gamma=900)$. The zero point energies (ZPE) of the pristine and $\mathrm{Cr}$ $\mathrm{Cr}, \mathrm{Cr}-\mathrm{Mn}, \mathrm{Cr}-\mathrm{Fe}$ co-doped grap-hene nanosheet are respectively $-7441.01 \mathrm{eV},-12049.24 \mathrm{eV}-10272.48 \mathrm{eV}$ and $-10447.82 \mathrm{eV}$.

From the values zero point energies (ZPE), it can be said that among the Cr-group co-doped structures, $\mathrm{Cr}$ $\mathrm{Cr}$ co-doped graphene nanosheet show better stability than others. 
Table 2: Bond lengths in angstrom $(\AA)$ for pristine graphene (PG), Cr-Cr, Cr-Mn and $\mathrm{Cr}-\mathrm{Fe}$ co-doped graphene nanosheet.

\begin{tabular}{|l|c|c|c|}
\hline Bond & $\begin{array}{c}\text { Cr-Cr } \\
\text { (TM1-TM2) }\end{array}$ & $\begin{array}{c}\text { Cr-Mn } \\
\text { (TM1-TM2) }\end{array}$ & $\begin{array}{c}\text { Cr-Fe } \\
\text { (TM1-TM2) }\end{array}$ \\
\hline C-C( PG) & 1.42 & 1.42 & 1.42 \\
\hline TM1-C6 & 1.69 & 1.68 & 1.71 \\
\hline TM1-C17 & 1.71 & 1.67 & 1.69 \\
\hline TM1-C21 & 1.69 & 1.67 & 1.69 \\
\hline TM2-C31 & 1.69 & 1.66 & 1.66 \\
\hline TM2-C35 & 169 & 1.66 & 1.66 \\
\hline TM2-C44 & 1.71 & 1.66 & 1.67 \\
\hline
\end{tabular}

\section{Electronic properties}

In solid-state physics, the band structure of a solid defines the span of energies that an electron within the solid may have which is called allowed energy bands and spans of energy that it may not have which is called band gaps or forbidden bands. Generally, there are three types of energy bands which are empty, filled, mixed or forbidden bands. The energetic levels are occupied by the electrons beginning with the lowest energy value level.

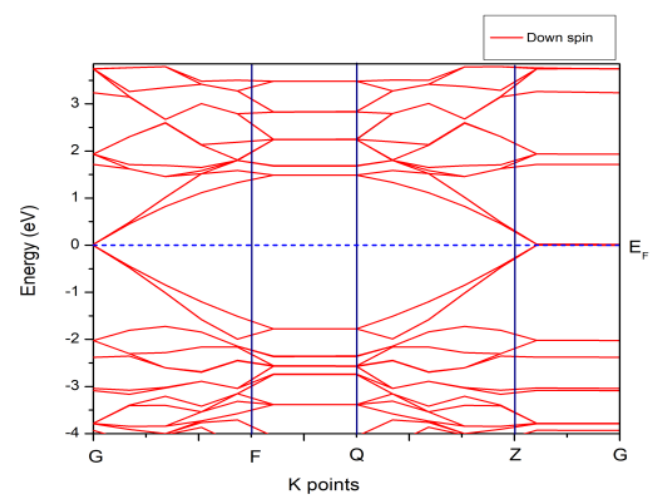

Pristine graphene

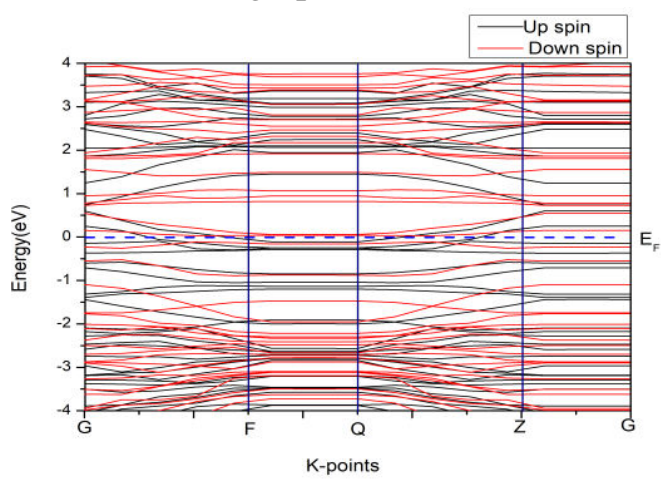

Cr-Mn co-doped
The electrons that engaged to the electrical conduction occupy the higher energy bands. The valence band relates to the highest energy band that holds electrons. The valence band can be completely or partial filled. The permitted states in the valence band add role to the electric current. The conduction band is the lowermost energetic band with unoccupied states. In materials, the conducting bands of empty, filled or allowed states can intervene with the forbidden bands and this is termed as band gap. Band gap refers to the energy change between the bottom point of the conduction band and the uppermost point of the valence band. The width of the band gap defines the category of material: insulator, semiconductor, metal. Any semiconductor either has a direct or an indirect band gap. Direct band gap can be described by having the band edges aligned in $\mathrm{k}$, so that there is a smooth transfer from the lowest state of valence band to the uppermost state of conduction band, with the emission of a photon, without fluctuating noticeably the momentum.
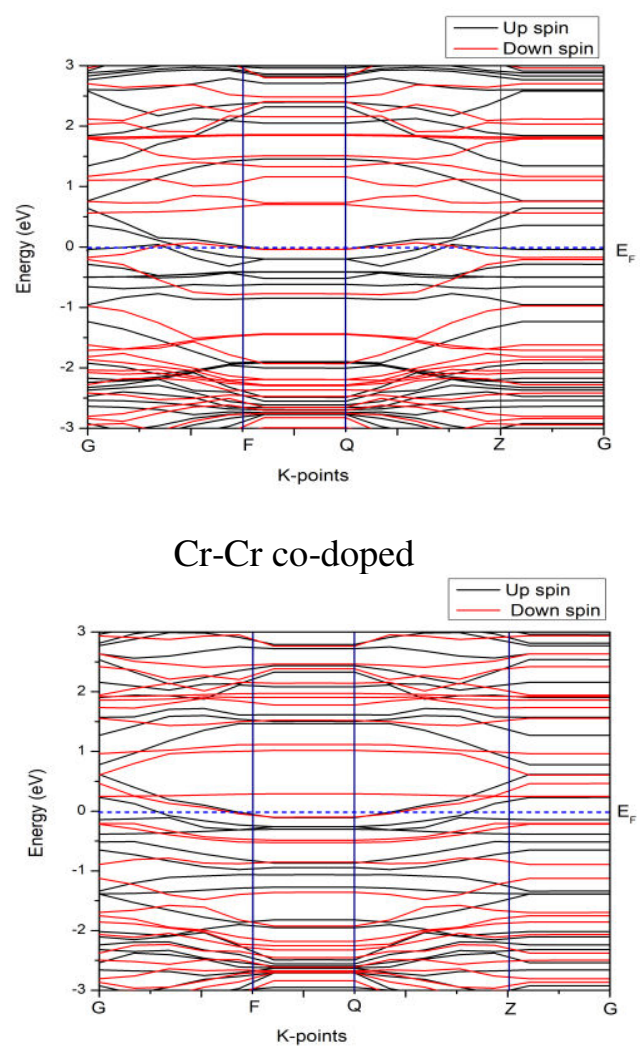

Cr-Fe co-doped

Fig 2: Band structures for pristine and $\mathrm{Cr}-\mathrm{Cr}, \mathrm{Cr}-\mathrm{Mn}, \mathrm{Cr}-\mathrm{Fe}$ co-doped graphene nanosheet. 
Conversely, in the indirect band gap the band edges are not aligned so the electron doesn't transfer exactly to the conduction band. In this method both a photon and a phonon are engaged. Band structures have been pictured in Fig 2 for pristine and Cr-group co-doped graphene nanosheet. For pristine graphene (Fig 2), the valence band maxima (VBM) and conduction band minima $(\mathrm{CBM})$ creates a cone which makes it a zero band gap (B.G) material and this cone is popularly known as Dirac cones. This unique character makes graphene more eye-catching for numerous applications. But when we doped TM pairs in the pristine graphene (PG), then we have surprisingly noticed that valence band overlapped the conduction band and they shows metallic nature for $\mathrm{Cr}-\mathrm{Cr}, \mathrm{Cr}-\mathrm{Mn}, \mathrm{Cr}-\mathrm{Fe}$ codoped graphene nanosheet. This fluctuation in the band signifies the faster transport of the photoelectrons and makes graphene useful in optical device. Also from all the co-doped nanosheets, it is found that they are all direct materials. Density of states (DOS) of a system is the number of different states at a particular energy level that are allowed to be occupied by electrons. Analysis of DOS plots is very common in materials science researches. Molecular DOS plots indicate the

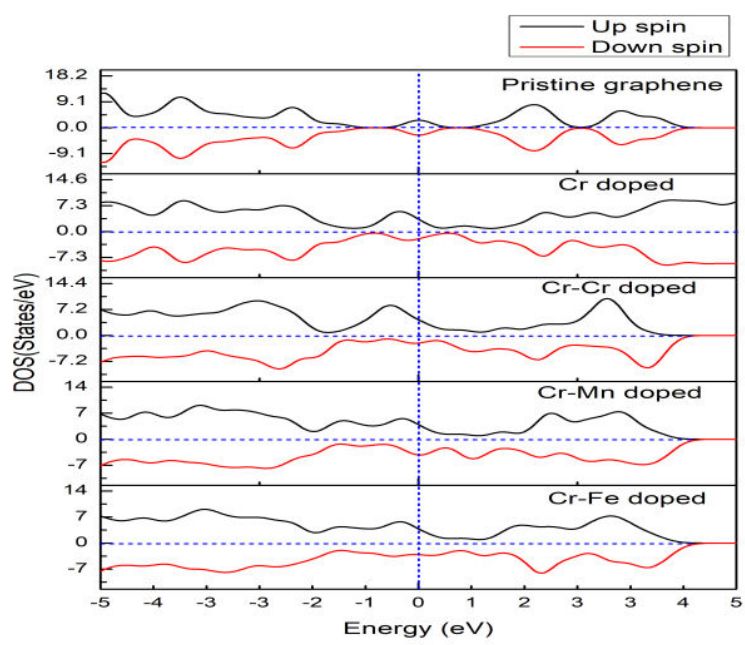

(a) occupancy of the electrons in different orbitals. Different properties especially electric and magnetic properties can be inferred from DOS plots ( $\operatorname{Rad}$ et al., 2016; Kittel, 2010; Harwood et al., 1998). To know the bonding mechanism among the atoms, we have further studied the DOS for all the doped structures. A comparison of total DOS plot among the pristine and TM co-doped graphene nanosheet are depicted in Fig 3.

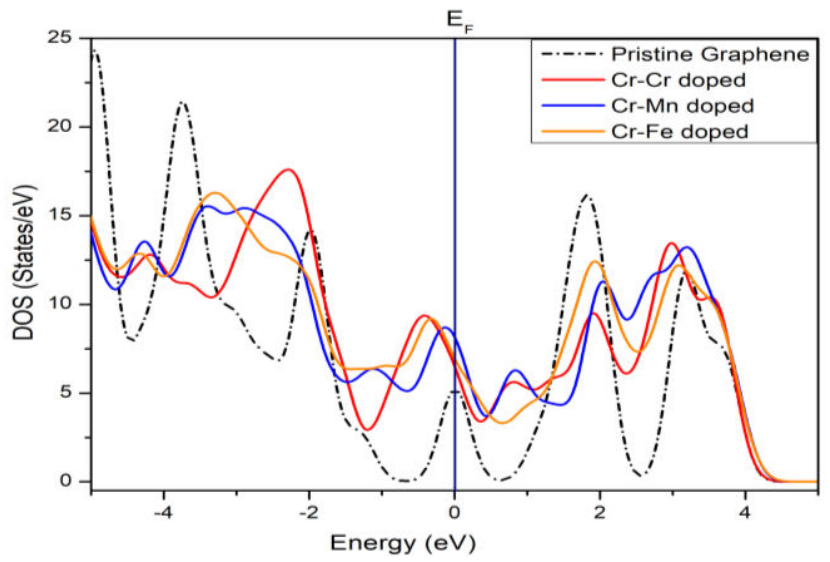

Fig 3: DOS of for pristine and $\mathrm{Cr}-\mathrm{Cr}, \mathrm{Cr}-\mathrm{Mn}, \mathrm{Cr}-$ $\mathrm{Fe}$ co-doped graphene nanosheet.

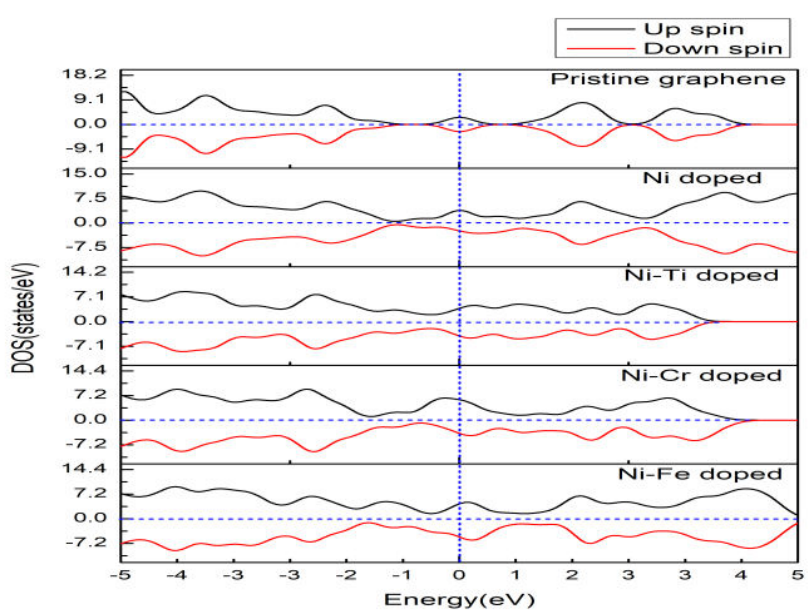

(b)

Fig 4: Spin polarized Density of states (DOS) of (a) pristine and Cr group co-doped graphene nanosheet and (b) pristine and Ni group co-doped graphene nanosheet.

In Fig 3, all the peak of the co-doped nanosheets shifted to the left from the Fermi level and in the conduction band peak amplitude decreases from $\mathrm{Cr}-\mathrm{Fe}$ to $\mathrm{Cr}-\mathrm{Cr}$ co-doped graphene nanosheet. Thus it is connected with the atomic radius of these doped atom and the valence $\mathrm{d}$ orbital electron which is ascending UniversePG I www.universepg.com from $\mathrm{Cr}$ to $\mathrm{Fe}$. We have further noticed that the valence band overlap the Fermi level and that's why no band gap has been found from the DOS structures. This is a reaffirmation of our study as similar pattern have been found in the band structures. 


\section{Magnetic properties}

For better understanding of the magnetic properties, we have calculated the spin polarized density of states for the investigating nanosheets and they are depicted in Fig 4. The ideal graphene is diamagnetic and for that reason no variation has been observed in the up and down of the spin polarized DOS. Theoretical (Yazyev \& Helm, 2007) and experimental ( Nair et al., 2012), (Ugeda et al., 2010) studied have shown that defects lead to increasing of magnetic moment of graphene. However, due to presence of defects, graphene show interacting magnetic moments and that's why we have seen variation between up and down spin in the $\mathrm{Cr}-\mathrm{Cr}, \mathrm{Cr}-\mathrm{Mn}$ and $\mathrm{Cr}-\mathrm{Fe}$ co-doped graphene nanosheet in Fig 4(a). These changes are related to increase of the unpaired electrons as doped TMs have unpaired $\mathrm{d}$ electrons. It is prominent for $\mathrm{Cr}-\mathrm{Cr}$ codoped graphene nanosheet at Fermi level which is set to zero as $\mathrm{Cr}$ has more unpaired $\mathrm{d}$ electrons as compared to the other TM atoms ( $\mathrm{Mn}$ and $\mathrm{Fe}$ ).

\section{Effect of Ni-3d TM co-doping in Graphene Nano- sheet}

\section{Structural Properties}

The optimized structures of Ni-Ti, Ni-Cr, Ni-Fe codoped graphene nanosheets and their electron density difference are shown in Fig 5. We have noticed that optimized pristine graphene and $\mathrm{Ni}-\mathrm{Ti}, \mathrm{Ni}-\mathrm{Cr}, \mathrm{Ni}-\mathrm{Fe}$ co-doped graphene nanosheets are perfect planer but in co-doped structures, bond lengths again found distorted around the TM atoms. Mulliken charge distribution has been calculated with the help of the same level of theory for the optimized pristine and $\mathrm{Ni}$ group co-doped graphene nanosheet. The data of Mul-liken charge transfer between the donors and acceptors are tabulated in Table 3.

Table 3: Mulliken charge transfer on pristine graphene (PG), Ni-Ti, Ni-Cr and Ni- Fe co-doped graphene nanosheet.

\begin{tabular}{|c|c|c|c|c|}
\hline Species & $\begin{array}{c}\text { Pristine } \\
\text { graphene }\end{array}$ & $\begin{array}{c}\text { Ni-Ti } \\
\text { (TM2-TM1) }\end{array}$ & $\begin{array}{c}\text { Ni-Cr } \\
\text { (TM2-TM1) }\end{array}$ & $\begin{array}{c}\text { Ni-Fe } \\
\text { (TM2-TM1) }\end{array}$ \\
\hline C6 & 0 & -0.39 & -0.30 & -0.34 \\
\hline C17 & 0 & -0.39 & -0.30 & -0.34 \\
\hline C21 & 0 & -0.39 & -0.30 & -0.34 \\
\hline C31 & 0 & -0.32 & -0.32 & -0.32 \\
\hline C35 & 0 & -0.32 & -0.32 & -0.32 \\
\hline C44 & 0 & -0.32 & -0.32 & -0.33 \\
\hline TM1 & - & 2.18 & 1.69 & 1.80 \\
\hline TM2 & - & 1.72 & 1.71 & 1.71 \\
\hline
\end{tabular}

UniversePG I www.universepg.com
In graphene sheet, every time we have replaced $\mathrm{C} 30$ by $\mathrm{Ni}$ atom and $\mathrm{C} 20$ is by $\mathrm{Ti}, \mathrm{Cr}$, and $\mathrm{Fe}$ atoms respectively. Here the position of $\mathrm{C} 20$ is considered as TM1 the position of C30 is TM2. For the Ni group codoped graphene nanosheet, $\mathrm{C}$ atoms show negative charges and TMs show positive charges which means that $\mathrm{C}$ atoms act as acceptors and TMs are donors.

As bond lengths and atomic distances in the nanosheet are directly reliant on the charge circulation of the entire system. All of the bond lengths and atomic distances for the Ni group co-doped graphene nanosheet are tabulated in Table 4. The zero point energies (ZPE) of the pristine and Ni-Ti, Ni-Cr, and Ni-Fe codoped graphene nanosheet are respectively -7441 . $01 \mathrm{eV},-10069.20 \mathrm{eV},-10936.87 \mathrm{eV}$ and $-9334 .-85 \mathrm{eV}$. From the values zero point energies (ZPE), it can be said that $\mathrm{Ni}-\mathrm{Cr}$ co-doped graphene nanosheet is more stable as compared to other structures in this group.

\section{Electronic properties}

Band structures and total DOS spectra of pristine and $\mathrm{Ni}$-group co-doped graphene nanosheet are presented in Fig 6. Here pristine graphene has no band gap but when we have doped TMs pair in the pristine graphene, then we have amazingly observed that valence band overlapped the conduction band and they shows metallic nature with a slight variation in $\mathrm{Ni}-\mathrm{Ti}$ doped graphene (B.G: $0.075 \mathrm{eV}$ ). This is quite negligible as it is too small to exhibit any specific property. As the valence electrons of the doped atom increases, they shifted right from the Fermi level as we have depicted in Fig 6(e). Also at the Fermi level, peak intensity increases as we go from $\mathrm{Ni}-\mathrm{Ti}$ to $\mathrm{Ni}-\mathrm{Fe}$ co-doped graphene as the atomic radius also increases respectively. In the conduction band, we have noticed a huge variations in the doped structures in comparison to the pristine graphene and again the valence band overlain the $\mathrm{E}_{\mathrm{F}}$ which validate our present study. $\mathrm{Cr}-\mathrm{Cr} \& \mathrm{Ni}-\mathrm{Cr}$ co-doped graphene nanosheet exhibit magnetic behavior which is suitable for magnetic device application.

\section{Magnetic properties}

Spin polarized density of states have in depicted in Fig 4(b) for Ni-group co-doped graphene nanosheet for the analysis of magnetic properties. As graphene does not show variation in their up and down spin but for TM co doping, we have found some variations. 
We have observed that $\mathrm{Ni}-\mathrm{Cr}$ co-doped graphene nanosheet exhibit better magnetic properties as it has shown more variation between up and down spin in the spin polarized density of states (DOS).

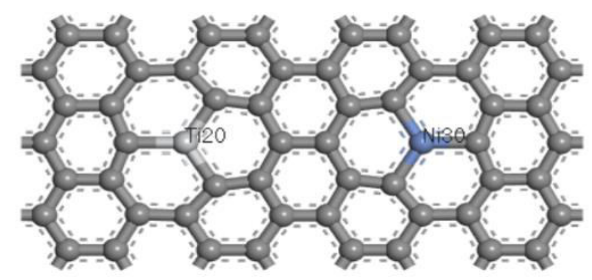

Optimized structure

\section{a. $\mathrm{Ni}$-Ti co-doped graphene nanosheet}

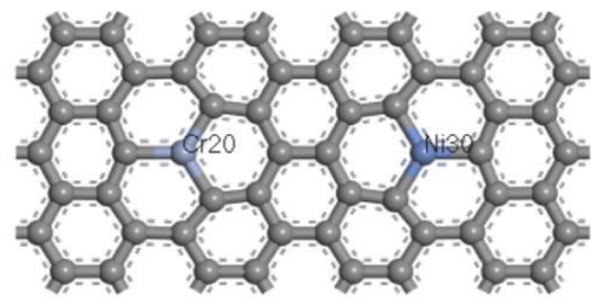

Optimized structure

\section{b. Ni-Cr co-doped graphene nanosheet}

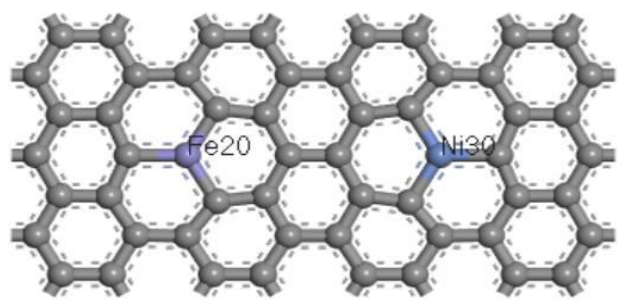

Optimized structure
This is very much favorable for their applications in various magnetic devices.

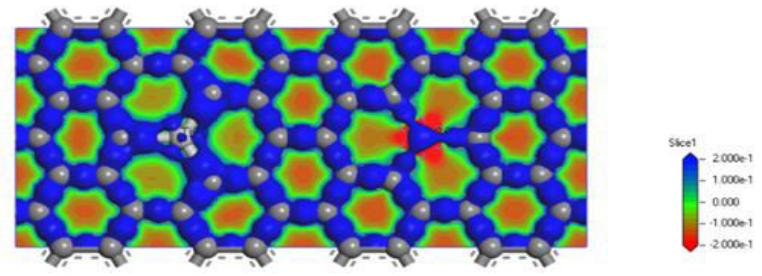

Electron density difference

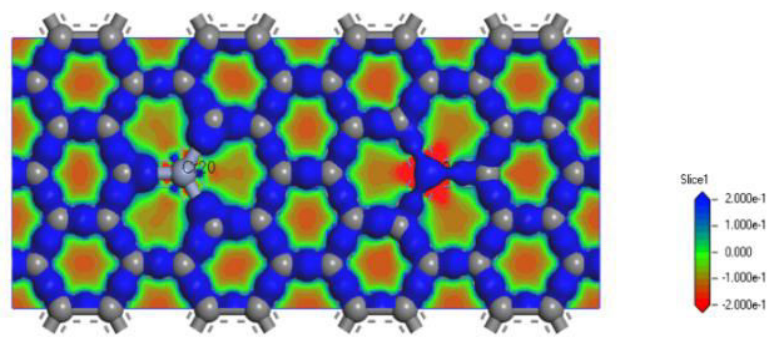

Electron density difference

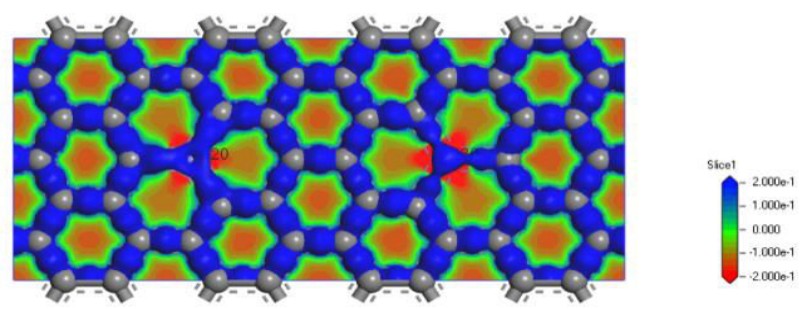

Electron density difference

\section{c. Ni-Fe co-doped graphene nanosheet}

Fig 5: Optimized structure and electron density difference of $\mathrm{Ni}-\mathrm{Ti}, \mathrm{Ni}-\mathrm{Cr}, \mathrm{Ni}-\mathrm{Fe}$ graphene nanosheet.

\section{CONCLUSION:}

In this article, we have theoretically investigated the structural, electronic and magnetic properties of pristine graphene and $3 \mathrm{~d}$ transition metal co-doped graphene nanosheet by using DFT framework. We have also analyzed the effect of TM co-doping in graphene nanosheet. From structural property, we have analyzed the Mulliken charge transfer, bond lengths, electron density difference and zero point energy for pristine graphene and $3 \mathrm{~d}$ transition metal co-doped graphene nanosheet. From the zero point energy, we can say that $\mathrm{Cr}-\mathrm{Cr}$ and $\mathrm{Ni}-\mathrm{Cr}$ doped graphene are more stable than other $\mathrm{Cr}$ - and $\mathrm{Ni}$ - co-doped structures. The zero point energies for $\mathrm{Cr}-\mathrm{Cr}$ and $\mathrm{Ni}-\mathrm{Cr}$ co-doped graphene are respectively $-12049.24 \mathrm{eV}$ and $-10936.87 \mathrm{eV}$. Band structure of TMs co-doped graphene nanosheet reveals metallic behaviors for all the co-doped structure except Ti-Ni which has shown a little band gap. Spin polarized Density of states (DOS) of $\mathrm{Cr}$ and $\mathrm{Ni}$ group indicate that $\mathrm{Cr}$ group co-doped graphene nanosheet show better magnetic properties. 


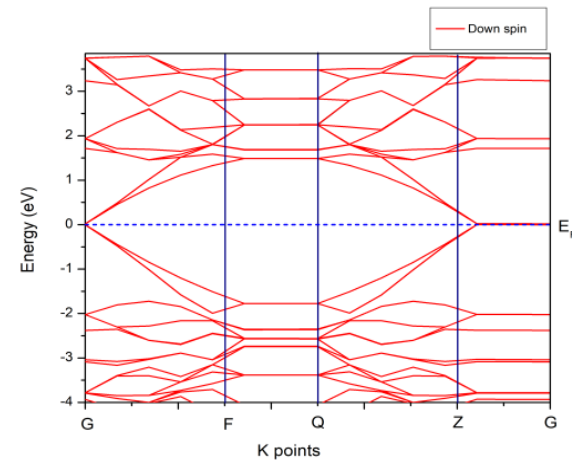

(a) Pristine graphene

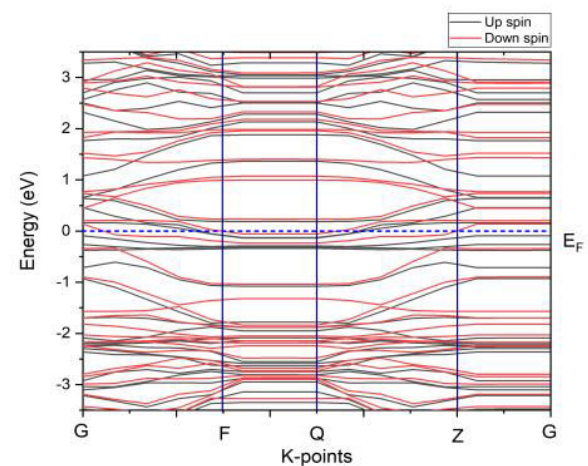

(c) Ni-Cr co-doped

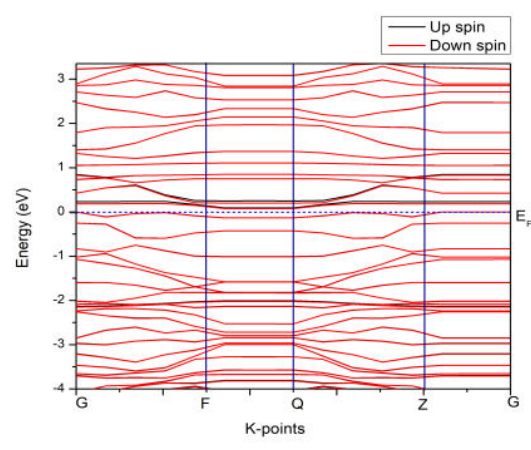

(b) Ni-Ti co-doped

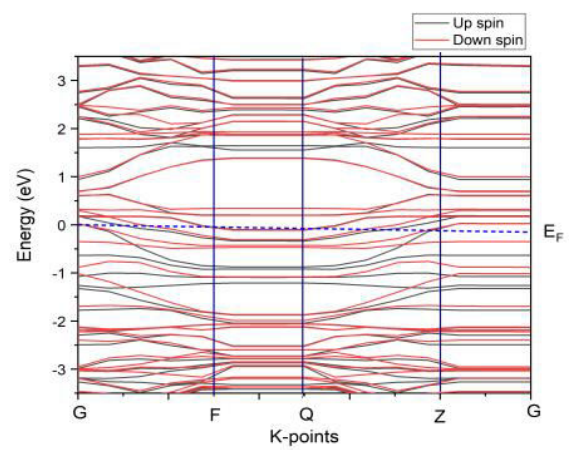

(d) Ni-Fe co-doped

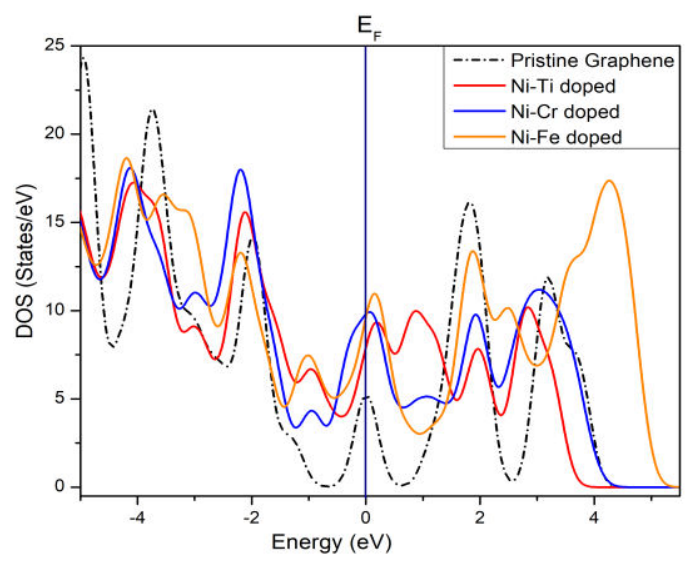

(e) Total DOS spectra

Fig 6: Band structures (a-d) and total DOS spectra (e) for pristine and $\mathrm{Ni}-\mathrm{Ti}, \mathrm{Ni}-\mathrm{Cr}, \mathrm{Ni}-\mathrm{Fe}$ co doped graphene nanosheet.

\section{ACKNOWLEDGEMENT:}

We are very thankful to PUST Research Cell for their fund allocation to setup the Computational Lab at the Department of Physics in Pabna University of Science and Technology for giving me opportunity to complete this work. We also thankfully acknowledge the Higher Education Quality Enhancement Program (HEQEP) subproject CP-3415, University Grant Commission (UGC) of Bangladesh, and the World Bank for the financial assistance to set up the Computational Physics (CP) Research Lab in the Dept. of Physics at Jahangirnagar University.

\section{CONFLICTS OF INTEREST:}

The authors declare that they have no known competing financial interests or personal relationships that could have appeared to influence the work reported in this paper. 


\section{REFERENCES:}

1) A. K. Rajagopal and J. Callaway, (1973). Inhomogeneous electron gas. Phys. Rev. B, 17(5), 1912-1919.

https://link.aps.org/doi/10.1103/PhysRevB.7.1912

2) A. S. Rad, (2016). Al-doped graphene as a new nanostructure adsorbent for some halomethane compounds: DFT calculations. Surf. Sci., 645, 612. https://doi.org/10.1016/j.susc.2015.10.036

3) A. Shokuhi Rad, M. Esfahanian, S. Maleki, and G. Gharati, (2016). Application of carbon nanostructures toward So2 and So3 adsorption: A comparison between pristine graphene and $\mathrm{N}$ doped graphene by DFT calculations. J. Sulfur Chem., 37(2), 176-188. https://doi.org/10.1080/17415993.2015.1116536

4) B. W. Alphenaar, K. Tsukagoshi, and M. Wagner, (2001). Spin transport in nanotubes (invited). $J$. Appl. Phys., 89(11 II), 6863-6867. https://doi.org/10.1063/1.1359220

5) C. Binns, S. H. Baker, S. N. Mozley, C. Norris, H. S. Derbyshire, and S. C. Bayliss, (1996). Morphology of islanded trans-ition-metal films on graphite studied by extreme-UV reflectivity. Phys. Rev. B, 53(11), 7451-7459. https://link.aps.org/doi/10.1103/PhysRevB.53.7451

6) Chen, J.H., Jang, C., Xiao, S., Ishigami, M. and Fuhrer, M.S., (2008). Intrinsic and Extrinsic Performance of Graphene Devices on SiO2. Nature Nanotechnology, 3, 206-209. https://doi.org/10.1038/nnano.2008.58

7) C. Kittel, (2010). Introduction to Solid State Physics, $8^{\text {th }}$ Edition. ISBN: 978-0-471-41526-8.

8) Daniel Wegner, Ryan Yamachika, Yayu Wang, Victor W. Brar, Bart M. Bartlett, Jeffrey R. Long, and Michael F. Crommie, (2008). Singlemolecule charge transfer and bonding at an organic/inorganic interface: Tetracyanoethylene on noble metals. Nano Lett., 8(1), 131-135. https://doi.org/10.1021/n1072217y

9) Geim, A.K. and Kim, P., (2008). Carbon Wonderland. Scientific American, 298 (4), 90-7. https://doi.org/10.1038/scientificamerican0408-90

10) H. Monkhorst and J. Pack, (1976). Special points for Brillouin zone integrations. Phys. Rev. B, 13 (12), 5188-5192. https://doi.org/10.1103/PhysRevB.13.5188
11) J. Perdew, K. Burke, and M. Ernzerhof, (1996). Generalized Gradient Approximation Made Simple. Phys. Rev. Lett., 77(18), 3865-3868. https://link.aps.org/doi/10.1103/PhysRevLett.77.38 65

12) L. Chen, R. Wu, N. Kioussis, and J. R. Blanco, (1997). First principles investigations of 4d magnetism on C (0001). J. Appl. Phys., 81(8), 41614163. https://doi.org/10.1063/1.365166

13) L. M. Harwood, C. J. Moody, and J. M. Percy, (1998). Experimental organic chemistry: standard and microscale. Exp. Org. Chem. Stand. microscale, $2^{\text {nd }}$ Edition. ISBN-13: 978-0632048199

14) M D Segall, Philip J D Lindan, M J Probert, C J Pickard, P J Hasnip, S J Clark and M C Payne, (2002). First-principles simulation: Ideas, illustrations and the CASTEP code. J. Phys. Condens. Matter., 14(11), 2717-2744. https://doi.org/10.1088/0953-8984/14/11/301

15) M. Menon, A. N. Andriotis, and G. E. Froudakis, (2000). Curvature dependence of the metal catalyst atom interaction with carbon nanotubes walls. Chem. Phys. Lett., 320(5), 425434.

16) M. M. Ugeda, I. Brihuega, F. Guinea, and J. M. Gómez-Rodríguez, (2010). Missing atom as a source of carbon magnetism. Phys. Rev. Lett., 104(9), 096804. https://doi.org/10.1103/PhysRevLett.104.096804

17) N. Gorjizadeh, A. A. Farajian, K. Esfarjani, and Y. Kawazoe, (2008). Spin and bandgap engineering in doped graphene nanoribbons. Phys. Rev. B - Condens. Matter Mater. Phys., 78(15), 155427. https://doi.org/\%2010.1103/PhysRevB.78.155427

18) O. V. Yazyev and L. Helm, (2007). Defectinduced magnetism in graphene. Phys. Rev. B Condens. Matter Mater. Phys., 75 (12), 125408. https://doi.org/10.1103/PhysRevB.75.125408

19) P. Avouris, (2010). Graphene: Electronic and Photonic Properties and Devices. Nano Letters, 10(11), 4285-4294. https://doi.org/10.1021/nl102824h

20) Pathiranage D. (2021). Numerical investigation of dropwise condensation on smooth plates with 
different wettability, Int. J. Mat. Math. Sci., 3(3), 60-73. https://doi.org/10.34104/ijmms.021.060073

21) Peter V.E. McClintock, (2012). Graphene: Carbon in Two Dimensions, by Mikhail I. Katsnelson. Contemporary Physics, 53(6), 503-504. https://doi.org/10.1080/00107514.2012.732963

22) R. R. Nair, M. Sepioni, I-Ling Tsai, O. Lehtinen, J. Keinonen, A. V. Krasheninnikov, T. Thomson, A. K. Geim, I. V. Grigorieva, (2012). Spin-half paramagnetism in graphene induced by point defects. Nat. Phys., 8(3), 199-202. http://dx.doi.org/10.1038/nphys2183

23) S. Nagao, T. Kurikawa, K. Miyajima, A. Nakajima, and K. Kaya, (1998). Formation and Structures of Transition Metal - C 60 Clusters. J. Chem. Phys., 4(98), 4495-4500.

https://doi.org/10.1021/jp981136a
24) Vanesa C. Sanchez, Ashish Jachak, Robert H. Hurt, and Agnes B. Kane, (2012).Biological Interactions of Graphene-Family Nanomaterials: An Interdisciplinary Review. Chemical Research in Toxicology, 25(1), 15-34.

https://doi.org/10.1021/tx200339h

25) W. Kohn and L. J. Sham, (1965). Self-consistent equations including exchange and correlation effects. Phys. Rev., 140(4A), A1133--A1138. https://link.aps.org/doi/10.1103/PhysRev.140.A1133

26) Y. Yagi, T. M. Briere, M. H. F. Sluiter, V. Kumar, A. A. Farajian, and Y. Kawazoe, (2004). Stable geometries and magnetic properties of single-walled carbon nano tubes doped with $3 \mathrm{~d}$ transition metals: A first-principles study. Phys. Rev. B, 69(7), 075414. https://doi.org/10.1103/PhysRevB.69.075414

Citation: Nishat M, Islam R, and Rahman MA. (2021). Investigation of the effect of 3d TM-TM atom co-doped in graphene nanosheet: DFT based calculations, Int. J. Mat. Math. Sci., 3(6), 122-132. https://doi.org/10.34104/ijmms.021.01220132 @) () 